\title{
Epidemiology and microbiology of invasive candidiasis in the largest tertiary Greek pediatric hospital during 2008-2017
}

\section{Noni ${ }^{1}$, A. Stathi ${ }^{2}$, I. Vaki ${ }^{1}$, L. Zachariadou ${ }^{2}$, E. Kyrikou ${ }^{2}$, A. Michos ${ }^{1}$}

${ }^{1} 1$ st Department of Pediatrics, "Aghia Sophia" Children's Hospital, Athens, Greece

${ }^{2}$ Department of Clinical Microbiology, "Aghia Sophia” Children's Hospital, Athens, Greece

\section{Background}

Candida species are a common cause of invasive infection in neonates and children. The aim was to study the epidemiology and microbiology of invasive candidiasis (IC) in the largest tertiary Greek pediatric hospital during a 10-year period.

\section{"Results}

*During a 10-year period, 178 incidences of invasive candidiasis were recorded." - Seventy-seven isolates (43.3\%) were detected in neonatal intensive care units (NICU), 46 (25.8\%) in pediatric clinics, 27 (15.2\%) in hematology/oncology units " (HOU) and 28 (15.7\%) in pediatric ICU (PICU).

* The tissue distribution included blood (87.1\%), cerebrospinal (7.9\%),

peritoneal (3.9\%) and pleural fluids (1.1\%).

* Ten different Candida species were detected. Candida albicans was the most

frequently isolated species $(47.8 \%)$ followed by $C$. parapsilosis $(28.7 \%), C$.

lusitaniae (6.2\%), C. glabrata (4.5\%) and C. tropicalis (4.5\%).

* The resistance in fluconazole was $0.0 \%$ among $C$. albicans isolates and $9.6 \%$ among C. parapsilosis isolates.

Table. Distribution of Candida species by infection site

\begin{tabular}{lcccc}
\hline Candida species & $\begin{array}{c}\text { Blood } \\
(\mathbf{n = 1 5 5})\end{array}$ & $\begin{array}{c}\text { CSF } \\
(\mathbf{n = 1 4})\end{array}$ & $\begin{array}{c}\text { Peritoneal fluid } \\
(\mathbf{n}=\mathbf{7})\end{array}$ & $\begin{array}{c}\text { Pleural fluid } \\
(\mathbf{n = 2})\end{array}$ \\
\hline Candida albicans $(\mathbf{n = 8 5})$ & $74(47.7)$ & $7(50.0)$ & $3(42.9)$ & $1(50.0)$ \\
Candida parapsilosis $(\mathbf{n = 5 1})$ & $45(29.0)$ & $3(21.4)$ & $3(42.9)$ & $0(0.0)$ \\
Candida lusitaniae $(\mathbf{n = 1 1})$ & $9(5.8)$ & $2(14.3)$ & $0(0.0)$ & $0(0.0)$ \\
Candida tropicalis $(\mathbf{n = 8})$ & $6(3.9)$ & $1(7.1)$ & $0(0.0)$ & $1(50.0)$ \\
Candida glabrata $(\mathbf{n = 8 )}$ & $8(5.2)$ & $0(0.0)$ & $0(0.0)$ & $0(0.0)$ \\
Candida famata $(\mathbf{n = 6})$ & $5(3.2)$ & $1(7.1)$ & $0(0.0)$ & $0(0.0)$ \\
Candida krusei $(\mathbf{n = 5})$ & $4(2.6)$ & $0(0.0)$ & $1(14.3)$ & $0(0.0)$ \\
Candida guilliermondii $(\mathbf{n}=\mathbf{2})$ & $2(1.3)$ & $0(0.0)$ & $0(0.0)$ & $0(0.0)$ \\
Candida rugosa $(\mathbf{n = 1})$ & $1(0.6)$ & $0(0.0)$ & $0(0.0)$ & $0(0.0)$ \\
Candida kefur $(\mathbf{n}=\mathbf{1})$ & $1(0.6)$ & $0(0.0)$ & $0(0.0)$ & $0(0.0)$ \\
\hline
\end{tabular}

\section{Methods}

-A retrospective cohort study was performed from January 2008 to

December 2017. Every first Candida isolation from each episode of IC was lincluded. Identification and susceptibility testing were performed according : to local procedures, while antifungal susceptibility testing was performed according to CLSI methodology.

Figure. Distribution of Candida species by year

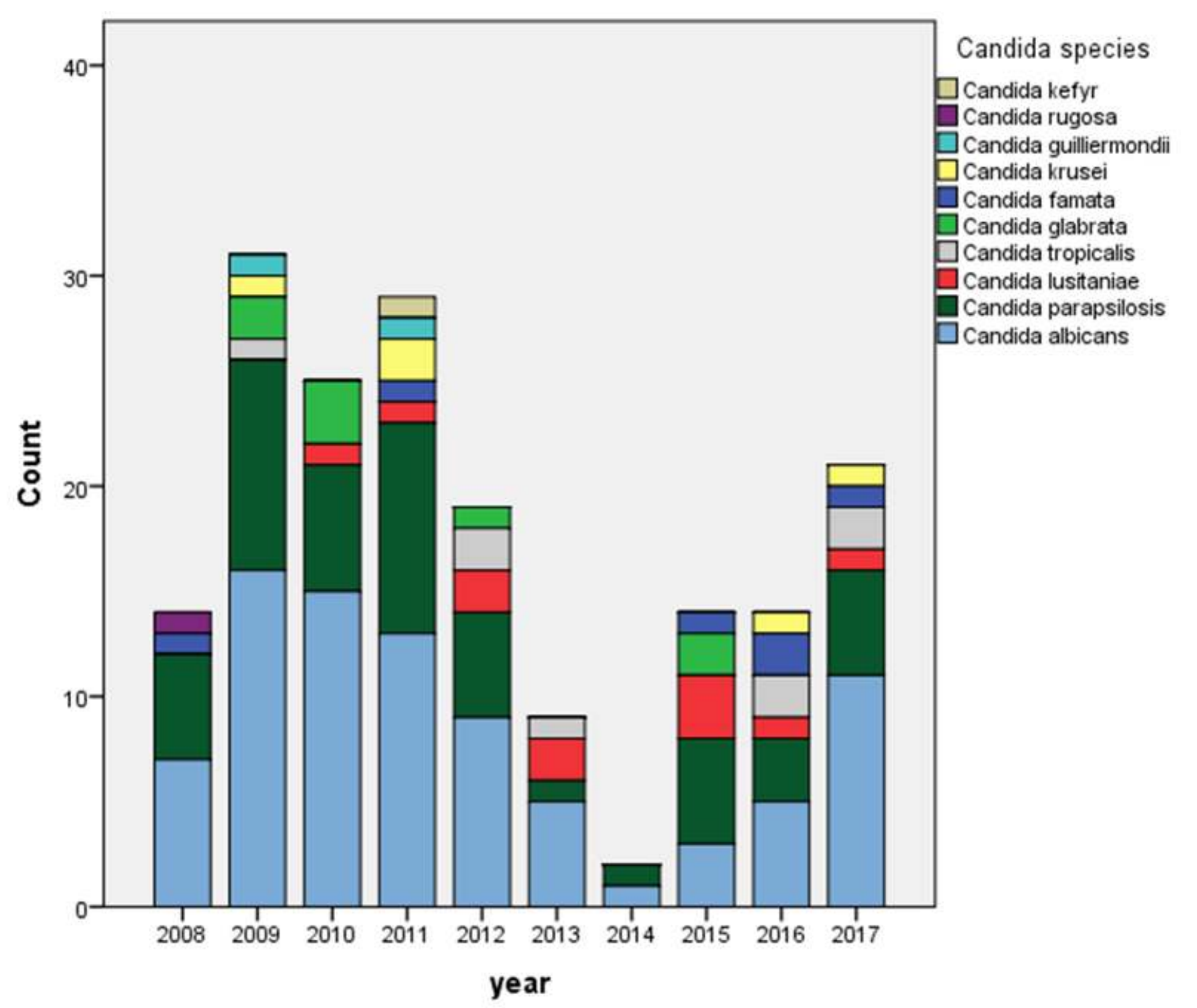

Conclusion

$>$ In our hospital, fluconazole sensitive C. albicans was the most frequently identified species compared with $C$. non-albicans.

" Local epidemiology monitoring is always necessary to understand invasive candidiasis burden in pediatric populations. 